\title{
ON A QUESTION OF REMESLENNIKOV
}

\author{
JAMES McCOOL* \\ Department of Mathematics, University of Toronto, Toronto, Ontario, Canada M5S 1A1 \\ e-mail:mccool@math.toronto.edu
}

(Received 18 June, 1999)

\begin{abstract}
We give an example of an element $r$ of a free group $F$, and an element $s$ of minimal length in the normal closure of $r$ in $F$, such that $s$ is not conjugate to $r^{ \pm 1}$ or to $\left[r^{ \pm 1}, f\right]$, for any element $f$ of $F$.
\end{abstract}

1991 Mathematics Subject Classification. 20E05, $20 \mathrm{~F} 05$.

1. Introduction. The question referred to in the title is Question F16 of the list 'Open problems in combinatorial group theory' of Baumslag, Myasnikov and Shpilrain [1], and it reads as follows.

Let $R$ be the normal closure of an element $r$ in a free group $F$ with the natural length function, and suppose that $s$ is a (non-identity) element of minimal length in $R$. Is it true that $s$ is conjugate to one of the following elements: $r, r^{-1},[r, f]$, or $\left[r^{-1}, f\right]$, for some element $f$ ?

In [1] it is noted that this question was motivated by a well known result of Magnus (see e.g. [2]): if elements $r$ and $s$ of a free group $F$ have the same normal closure, then $s$ is conjugate to $r^{ \pm 1}$. We would add that no general result classifying elements of (relatively) small length in the normal closure of a single element of a free group is known, and that such a result would be of great interest in the theory of one-relator groups.

The question is known to have a positive answer in a number of cases. Thus, for example, if $r$ satisfies a suitable small cancellation condition (see Chapter V of [2]), then it is easily seen (e.g. by using a theorem of Greendlinger; see Theorem 4.5 of Chapter $\mathrm{V}$ of [2]) that any element of minimal length in $R$ is conjugate to $r^{ \pm 1}$, while in the free group $F_{2}$ with basis $\{a, b\}$, if $r=a^{t} b^{2}$ and $t \geq 5$, it can be easily shown, using arguments similar to those given below, that the elements of minimal length in $R$ are conjugates of $\left[a^{ \pm 1}, b^{2}\right]$, and these are the same as conjugates of $\left[r^{ \pm 1}, a^{-1} r\right]$.

Let $r$ be the element of $F_{2}$ given by $r=b a^{t} b^{2} a^{t}$, where $t \geq 3$, and let $s=\left[b^{3}, a\right]$. We shall provide a negative answer to Remeslennikov's question by showing that $s$ is of minimal length in $R$, and that $s$ is not conjugate to $r^{ \pm 1}$ or $\left[r^{ \pm 1}, f\right]$, for any $f$ in $F_{2}$.

2. The proof. Let $G$ be the free product with amalgamation of two infinite cycles given by the presentation $\left\langle x, a \mid x^{3}=a^{-t}\right\rangle$. We have

$$
\begin{aligned}
G & =\left\langle x, a, b \mid x^{3}=a^{-t}, b=x^{2}\right\rangle=\left\langle x, a, b \mid b=x^{2}, x^{-1} b^{2}=a^{-t}\right\rangle \\
& =\left\langle a, b \mid b=b^{2} a^{t} b^{2} a^{t}\right\rangle=\left\langle a, b \mid b a^{t} b^{2} a^{t}=1\right\rangle .
\end{aligned}
$$

* Research supported by a grant from NSERC Canada. 
We note that $s \in R$ (since $b^{3}=x^{6}$ is central in $G$ ), $s$ is cyclically reduced, and $s$ is not conjugate to $r^{ \pm 1}$. We now check that $s$ is not conjugate to any element $\left[r^{ \pm 1}, f\right]$ of $F_{2}$. If it were conjugate to such a commutator, say to $\left[r^{-1}, f\right]=r^{-1} f r f^{-1}$, then we have $f r f^{-1}=f_{1} r_{1} f_{1}^{-1}$ say, where $r_{1}$ is a cyclic permutation of $r$, and no cancellation occurs in $f_{1} r_{1} f_{1}^{-1}$. We have $s$ is conjugate to $f_{1}^{-1} r^{-1} f_{1} r_{1}$, and we see that $f_{1}$ must be cancelled completely by $r^{-1}$, since otherwise $f_{1}^{-1} r^{-1} f_{1} r_{1}$, when reduced, would be cyclically reduced, but would have length greater than the length of $r$, and so could not be conjugate to $s$. Thus some cyclic permutation $s_{1}$ of $s$ is the product $r_{2}^{-1} r_{1}$, for certain cyclic permutations $r_{1}, r_{2}$ of $r$. We distinguish seven types of cyclic permutations of $r$, namely:

$$
b a^{t} b^{2} a^{t}, a^{t} b^{2} a^{t} b, a^{l_{2}} b^{2} a^{t} b a^{l_{1}}, b^{2} a^{t} b a^{t}, b a^{t} b a^{t} b, a^{t} b a^{t} b^{2}, a^{m_{2}} b a^{t} b^{2} a^{m_{1}},
$$

where $l_{1}+l_{2}=m_{1}+m_{2}=t$ and $l_{1}, l_{2}, m_{1}, m_{2}$ are positive. It is now easy to check that no product $r_{2}^{-1} r_{1}$ (or $r_{1} r_{2}^{-1}$ ) has length as small as 8 , except in the case $t=3$, and in case $t=3$ the only such products of length as small as 8 are cyclic permutations of $\left[b, a^{t}\right]$. Thus $s$ is not conjugate to any element of the form $\left[r^{ \pm 1}, f\right]$.

It remains to show that no non-identity element $w$ of $R$ could have length less than 8. Suppose that such a $w$ exists. We may assume that $w$ is of the form $b^{l_{1}} a^{m_{1}} \ldots b^{l_{k}} a^{m_{k}}$, where the $l_{i}, m_{i}$ are non-zero, and $\left|l_{1}\right|+\left|m_{1}\right|+\ldots+\left|l_{k}\right|+\left|m_{k}\right|<8$. We have $w=1$ in $G$. Replacing $b$ by $x^{2}$, we see, since $G$ is a free product with amalgamation, that either some $l_{i}$ is a multiple of 3 , or some $m_{i}$ is a multiple of $t$. Suppose that the former occurs. There is no loss of generality in supposing that $l_{1}$ is a positive multiple of 3 (replacing $w$ by a cyclic permutation of $w^{ \pm 1}$ if necessary ). If $l_{1}>3$, then $w$ must be $b^{6} a^{ \pm 1}$; but $b^{6} a^{ \pm 1} \neq 1$ in $G$. Thus we have $l_{1}=3$, and $w=b^{3} a^{m_{1}} b^{l_{2}} a^{m_{2}}$, where each $l_{i}$ and $m_{i}$ must have absolute value no more than 2 ; replacing $b$ by $x^{2}$ we see that such a $w$ is not the identity in $G$. Thus we have obtained a contradiction if some $l_{i}$ is a multiple of 3 . It is likewise easy to obtain a contradiction under the assumption that some $m_{i}$ is a multiple of $t$. This proves our claim that $s$ is of minimal length in $R$.

\section{REFERENCES}

1. G. Baumslag, A. G. Myasnikov and V. Shpilrain, Open problems in combinatorial group theory, World-wide-web: http://zebra.sci.ccny.cuny.edu/web/

2. R. C. Lyndon and P. E. Schupp, Combinatorial group theory, Ergebnisse der Mathematik, Band 189 (Springer-Verlag, 1977). 